\title{
Magnetic carbon spheres and their derivatives combined with printed electrochemical sensors
}

\author{
Stefano Cinti ${ }^{\text {a, }}$, Francesca Limosani ${ }^{\text {a, }}{ }^{\text {, }}$ Manuela Scarselli ${ }^{\mathrm{b}}$, Fabiana Arduini ${ }^{\mathrm{a}, *}$ \\ a Department of Chemical Science and Technologies, University or Rome "Tor Vergata", Via della Ricerca Scientifica 1, 00133 Rome, Italy \\ b Department of Physics, University of Rome "Tor Vergata", Via della Ricerca Scientifica 1, 00133 Rome, Italy
}

\section{A R T I C L E I N F O}

\section{Article history:}

Received 14 March 2018

Received in revised form

3 June 2018

Accepted 6 June 2018

Available online 7 June 2018

Keywords:

Magnetic carbon structures

Screen-printed electrodes

Smart sampling

\begin{abstract}
A B S T R A C T
Herein, we report the synthesis of several magnetic carbon structures starting from spheres (CSs) to more elongated structures to get tubes and fibers by customizing chemical vapour deposition parameters. These CSs-based products, ranging from nano-to micro-size, were investigated by morphological point of view using scanning electron microscopy, energy dispersive $\mathrm{x}$-ray spectroscopy, and Raman spectroscopy. These carbon structures were combined with screen-printed electrodes highlighting their electrochemical effectiveness towards the detection of several species, i.e. ferricyanide, ascorbic acid, dopamine, cysteine, serotonin, and NADH. The presence of iron nuclei within the carbonaceous lattices, besides having improved the electrochemical performances at the printed electrodes, might confer these CSs-based structures a future application in the field of remediation/sensing. Herein, some preliminary applications were provided showing that these materials can be easily employed to collect species and, successively, coupled with printed electrochemical sensors.
\end{abstract}

(c) 2018 Elsevier Ltd. All rights reserved.

\section{Introduction}

After the discovery of fullerenes, the research activity has been focused on the possibility of synthesizing other small size carbonaceous materials of variable shape. Among these, there are spheres, tubes, fibers, and more recently graphene, all ranging in the 'nano' regime dimension [1-3]. Beyond basic research, most of the success reached in practical applications relies on the good control gained over the structural and morphological properties of the final carbon products. Consequently, carbon-based nano-and micro-structures are now used as smart materials in several applications such as catalysis, energy storage, renewable energy, remediation, sensing, nanomedicine, and others [4-6]. Graphene and carbon nanotubes (CNTs) are the most employed materials thanks to their unique electric and mechanical properties, while applications of close-curved nanostructures (e.g. fullerenes) are more limited [7,8]. The carbon spheres (CSs) are closed shell carbon-based material curved as fullerenes, but they differ from the fullerene's family since their inner structure is constituted of numerous graphitic sheets that can be found in ordered or

\footnotetext{
* Corresponding author.

E-mail address: fabiana.arduini@uniroma2.it (F. Arduini).

1 These authors contributed equally.
}

disordered arrangement forming, in this last case, unclosed shells that eventually create defective reaction sites. Recently, there has been a renewed interest in CSs applications in electroanalytical field since they display good chemical stability, low density, high surface-to-volume ratio, and interesting electrochemical properties [9-12]. However, their better-known "brothers" i.e. graphene and nanotubes (single and multi-walled) often hide the effectiveness of CSs. Herein, we highlight the advantages of CSs-based materials to be exploited as electrochemical booster for screen-printed electrodes (SPEs). To produce carbonaceous materials with different shaped structures, Chemical Vapour Deposition (CVD) was employed as the technique; in fact by selecting some experimental parameters during CVD, i.e. gas flux, catalyst concentration, flow rate and duration, different structures can be easily realized. In this work, four different types of configured CSs-based structures were synthesized: spheres, aggregates, necklaces, and tubes. To evaluate their electrochemical performances, SPEs were modified with stable dispersions of the synthesized CSs-based structures and these miniaturized tools were tested in cyclic voltammetry using ferro/ ferricyanide as redox probe. In addition, the electroanalytical features of the various carbon structures, ranging from spheres (zero dimensional) to one dimensional (tubes) configurations [13], were also evaluated towards the detection of clinically relevant molecules, i.e. ascorbic acid, dopamine, cysteine, serotonin, NADH, 
highlighting a clear enhancement if compared with the bare SPEs.

Besides the intrinsic electrochemical effectiveness of these structures, their magnetic nature makes them suitable for application in the field of sensing and remediation. In principle, the magnetic carbon-based structures might be adopted to capture target analytes (or pollutants) and successively to detect those. This approach offers a step-forward for future applications, especially towards the reduction of the operative extra-tasks at the end-user stage. In fact, one might imagine that the end-user could use these materials to sample the analyte, even in a complex matrix, just by magnetically collecting it, followed by the measurement of the "sampled" analyte by means of whichever device. This approach was demonstrated through preliminary efforts: these magnetic CSs-based materials were easily employed to sample species and, successively, coupled with electrochemical devices, revealing the presence of the "sampled" species. As an outlook, the use of nonsophisticated synthetic protocols allows for producing smart platforms that can be easily functionalized with enzyme, antibody, and nucleic acids, for the development of CSs-based fully-enclosed bioassays.

\section{Experimental}

\subsection{Materials}

All the reagents were of analytical grade and they were purchased from Sigma Aldrich (USA).

\subsection{Screen-printed electrode fabrication}

SPEs was produced with a 245 DEK (Weymouth, UK) screenprinting machine. Graphite-based ink (Electrodag $423 \mathrm{SS}$ ) from Acheson (Milan, Italy) was used to print both the working and auxiliary electrode. Silver/silver chloride ink (Electrodag 477 SS) was used to print the pseudo-reference electrode. Flexible polyester film (Autostat HT5) purchased from Autotype Italia (Milan, Italy) was used as substrate. The diameter of the working electrode was $0.3 \mathrm{~cm}$ resulting in a geometric area of $0.07 \mathrm{~cm}^{2}$.

\subsection{Synthesis of CSs-based structures}

All carbon-based structures were produced by using a floating catalyst. CVD was carried out in a quartz tube inserted in a furnace at 760 Torr pressure keeping the temperature at $800^{\circ} \mathrm{C}$. In particular, a solution of variable concentration (see Table 1 ), containing ferrocene dissolved in 1,2-dichlorobenzene was continuously injected (at a constant rate) with a glass syringe and carried into the hot reaction zone by a combined acetylene/argon gas flux. At the end of the synthesis process, the reaction products were collected from the quartz tube and used without post-growth purification. Table 1 reports the values of the growth parameters adopted for each sample produced, including sccm (standard cubic centimeters per minute) which quantify the gas-flux.

Table 1

Detailed list of the sample preparation components used in the synthesis of the carbon nanostructures.

\begin{tabular}{lccc}
\hline Sample & $\mathrm{Ar} / \mathrm{C}_{2} \mathrm{H}_{2}[\mathrm{sccm}]$ & Concentration of solution $[\mathrm{M}]$ & Rate $[\mathrm{mL} / \mathrm{h}]$ \\
\hline Sphere & $50 / 25$ & 0.21 & 3 \\
Aggregate & $250 / 40$ & 0.27 & 4 \\
Necklace & $300 / 100$ & 0.27 & 4.5 \\
Tube & $500 / 100$ & 0.19 & 6.2 \\
\hline
\end{tabular}

\subsection{Procedure for preparation of CSs-dispersions}

$10 \mathrm{mg}$ of the selected powder were firstly dissolved in $5 \mathrm{~mL}$ of $\mathrm{N}, \mathrm{N}$-dimethylformamide (DMF), and subsequently $5 \mathrm{~mL}$ of distilled water were added. The mixture was then sonicated for $1 \mathrm{~h}$ at $59 \mathrm{kHz}$. The dispersion was stored in the dark at room temperature.

\subsection{Procedure for SPE modification}

SPEs were modified by drop casting. $6 \mu \mathrm{L}$ of the dispersion were drop cast onto the working electrode surface via three following steps of $2 \mu \mathrm{L}$. The modified SPEs were tested after the evaporation of the solvent dispersion.

\subsection{Amperometric study}

The amperometric study was carried out by measuring ascorbic acid using SPEs modified with different CSs-based structures namely spheres, aggregates, necklaces, and tubes. In detail, amperometric batch analysis was performed in $10 \mathrm{~mL}$ of stirred $0.05 \mathrm{M}$ phosphate buffer containing $0.1 \mathrm{M}$ potassium chloride $(\mathrm{pH}$ 7.4) with a selected applied potential of $0.15 \mathrm{~V} \mathrm{vs} \mathrm{Ag} / \mathrm{AgCl}$. When a stable baseline current was reached, the analyte was added and the response was recorded.

\section{Results and discussion}

\subsection{CSs-based structures characterization}

In this work, we synthesized several CSs-based structures starting from zero dimensional material i.e. spheres to one dimensional material i.e. tubes by CVD, as described in the experimental section. SEM analysis was performed to investigate the morphology of the obtained carbon-based products. Fig. 1 reports a collection of images collected from the four different growth conditions, showing variable final products namely sphere, aggregate, necklace, and tubes. As reported in Fig. 1A, the sphere structure is perfectly shaped and consists of individual particles, characterized by a mean diameter that ranges between $200 \pm 10 \mathrm{~nm}$. The increase of both the ferrocene and the gas rate favoured the accretion of the spheres with a mean diameter of $175 \pm 10 \mathrm{~nm}$ (Fig. 1B) as evaluated from a statistical analysis obtained by the TEM studies reported in Ref. [14]. The subsequent increase of the gas rate in the CVD chamber allowed for generating a more complex structure, named necklace where the spheres partially welded forming long arrangements characterized by a high degree of surface roughness (Fig. 1C). These necklace structures have a mean diameter of $1.4 \pm 0.2 \mu \mathrm{m}$ with a length that can reach $20 \mu \mathrm{m}$, although they show a wide variety of outer diameter and most of them are full. Some tubes exhibit the so-called Bamboo-shape and others are covered by carbon aggregates almost spherical in shape that increase the outer surface roughness. A statistical analysis gives an average diameter of $1.5 \pm 0.3 \mu \mathrm{m}$, and a length longer than $30 \mu \mathrm{m}$ (Fig. 1D). In addition, some of these structures showed a magnetic response and this behaviour can be ascribed to the presence of iron that is the only magnetic element made available in the reaction process [14]. X-Ray electron diffraction spectra (EDX) obtained on the sample denoted as aggregate shows the contribution of carbon, oxygen and iron, Fig. 2a. The aluminium signal comes from the sample holder.

The graphitic nature of these carbon materials was investigated using the Raman spectroscopy (Fig. 2b). In particular, we focused on the D-band and G-band. The D-band arises from aromatic rings while the G-band originates from the vibration of $\mathrm{sp}^{2}$-bonded carbon atom in a two-dimensional hexagonal lattice and represents 

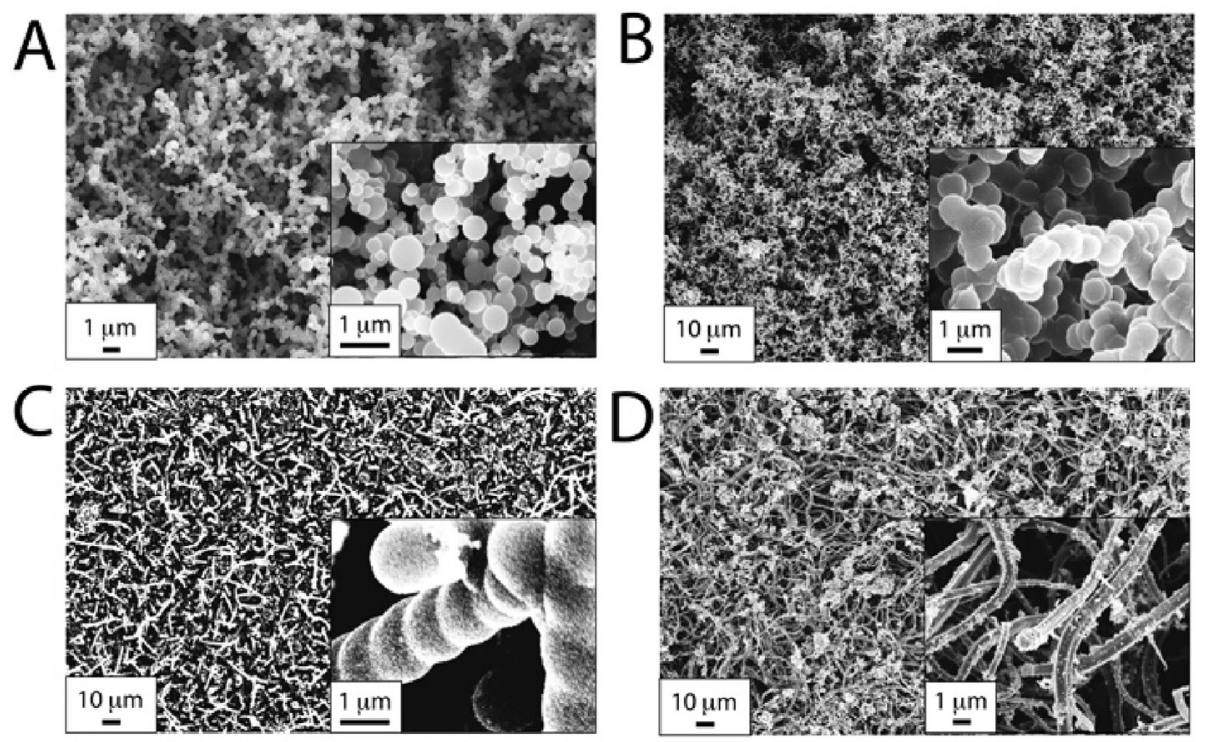

Fig. 1. SEM micrographs of A) sphere, B) aggregate, C) necklace, and D) tube structures.

a

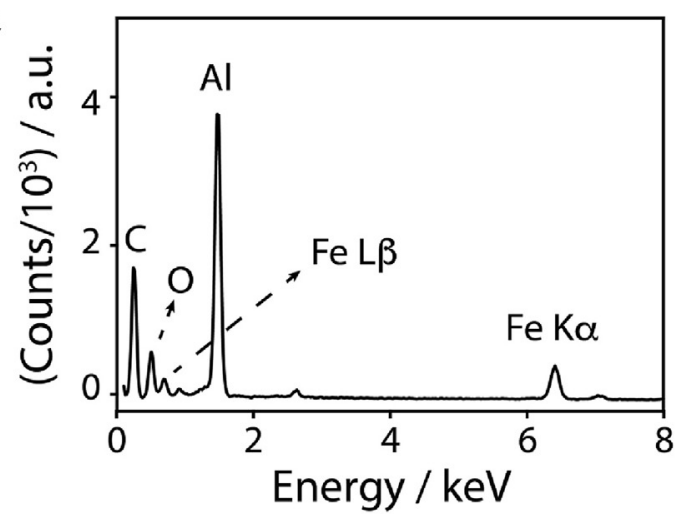

b

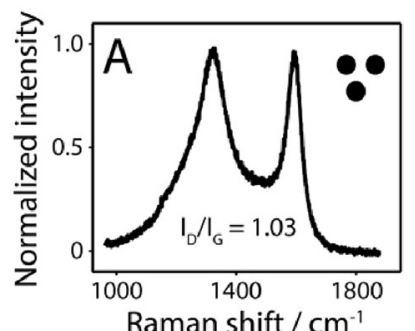

Raman shift $/ \mathrm{cm}^{-1}$
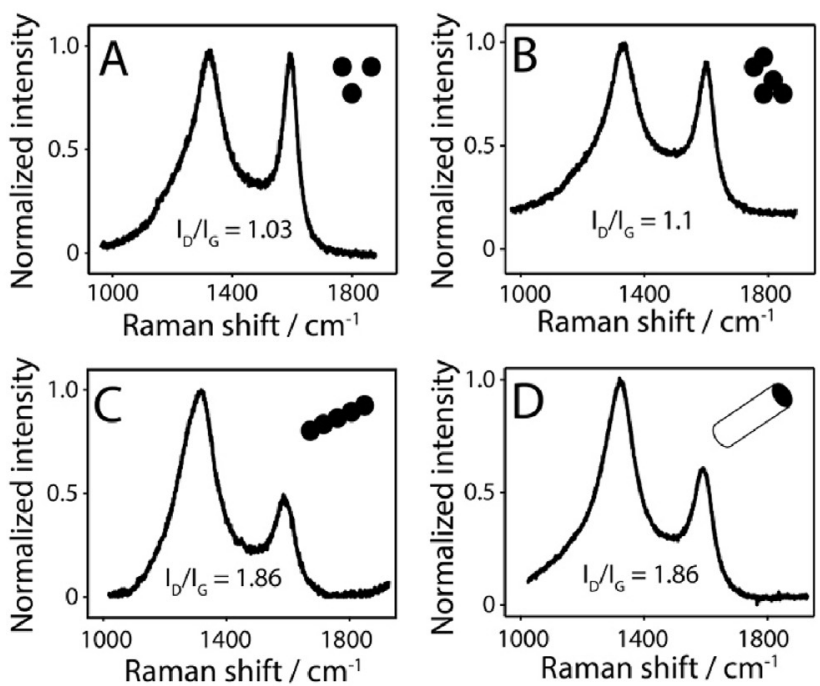

Fig. 2. a) EDX spectra of the magnetic aggregate structures, demonstrating the presence of Fe. b) Raman spectra of A) sphere, B) aggregate, C) necklace, and D) tube-based structures. the extent of graphitization. The structural quality was also analyzed from the $\mathrm{I}_{\mathrm{D}} / \mathrm{I}_{\mathrm{G}}$ ratio. This parameter is widely used as a measure of the size of the $\mathrm{sp}^{2}$ ring clusters in a network of $\mathrm{sp}^{3}$-and $\mathrm{sp}^{2}$-bonded carbons, giving information of structural distortions. The obtained values for the four samples range between 1.03 and 1.86. In particular, the lower value of 1.03 and 1.1 was found for the samples A and B, respectively. This ratio values are comparable to those reported in the literature for similar but no magnetic CSs $[15,16]$. The intensity of the D- and G-bands is comparable for samples $\mathrm{A}$ and $\mathrm{B}$, highlighting the presence of structural imperfections. The imperfections can be originated from the disorder in the graphene sheets relative orientation in the spheres as well as from the presence of pentagon and heptagon carbon rings that are necessary to bend the graphene during the spheres formation. Nonetheless, both the $D$ and $G$ peaks are sharp, suggesting the presence of mostly $\mathrm{C} \mathrm{sp} \mathrm{p}^{2}$ hybridization form in the nanostructure.

The samples $C$ and $D$ have similar spectra that show a marked contribution from the D-band with respect to the G-band with a measured $\mathrm{I}_{\mathrm{D}} / \mathrm{I}_{\mathrm{G}}$ ratio of 1.55 and 1.86 , respectively.

The G-band of these two samples decreases in intensity and broadens. It can be noticed, that the D-band for the four samples has always a marked intensity, due to the disorder and the change in the size distribution of the carbon nano-structures in the assembly.

\subsection{Electrochemistry of the CSs-based structures}

\subsubsection{Functionalization of the printed platforms}

In order to characterize the CSs-based structures by an electrochemical point of view, the printed electrodes were modified by drop casting method with CSs dispersions (Fig. 3). Briefly, all structures were dispersed in a solution made by $50 \% \mathrm{v} / \mathrm{v}$ water/ dimethylformamide, as previously reported for other carbon-based modifiers [17,18], and SPEs were modified with these different dispersions (sphere, aggregate, necklace, tube) via three following steps of $2 \mu \mathrm{L}$, with the aim to custom an easy and automatable way for miniaturized printed sensor manufacturing.

\subsubsection{Heterogeneous electron transfer constant evaluation}

To evaluate the electrochemical features of the different shaped 


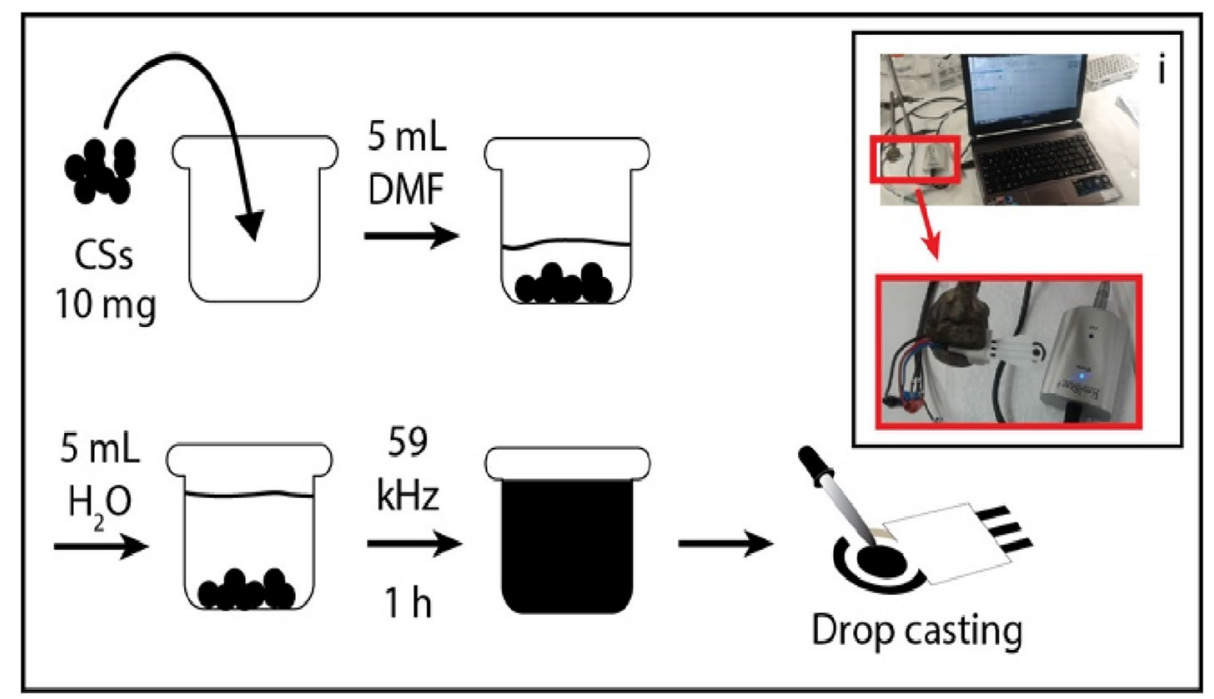

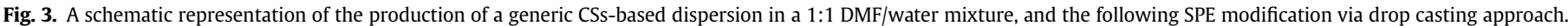
Inset: a photographic image of the experimental setup and a major focus on the SPE and the small potentiostat used to perform the experiments.

materials, heterogeneous electron transfer constant $\left(\mathrm{k}^{0}\right)$ was calculated using the inner-sphere ferro-/ferricyanide redox couple $[19,20]$ and Nicholson method [21]. In detail, the following equation: $\mathrm{k}^{0}=\Psi\left[\mathrm{D}_{\mathrm{O}} \pi v(\mathrm{nF} / \mathrm{RT})\right]^{1 / 2} /\left(\mathrm{D}_{\mathrm{O}} / \mathrm{D}_{\mathrm{R}}\right)^{\alpha / 2}$ was employed, where $\Psi$ is the kinetic parameter that is dependent on the separation between anodic and cathodic peaks, $\Delta \mathrm{E}, \mathrm{D}_{\mathrm{R}}$ and $\mathrm{D}_{\mathrm{O}}$ represent the diffusion coefficients of the reduced and the oxidized specie, $v$ is the scan rate, $\mathrm{F}$ is the Faraday constant, $\alpha$ is the transfer coefficient (0.5), and $\mathrm{n}$ is the number of electrons transferred in the electrochemical process. In this regard, we obtained $\mathrm{k}^{0}$ equal to $(7.7 \pm 1.3) 10^{-3}$, $(6.0 \pm 0.2) 10^{-3},(9 \pm 1) 10^{-3},(5 \pm 1) 10^{-3} \mathrm{~cm} / \mathrm{s}$ for sphere, aggregate, necklace, and tube structures, respectively. Furthermore, due to the high peak-to-peak separation of the unmodified SPE, it was not possible to calculate the $\mathrm{k}^{0}$ for bare SPE, highlighting the improvement of the electron transfer using SPEs modified with these carbon structures.

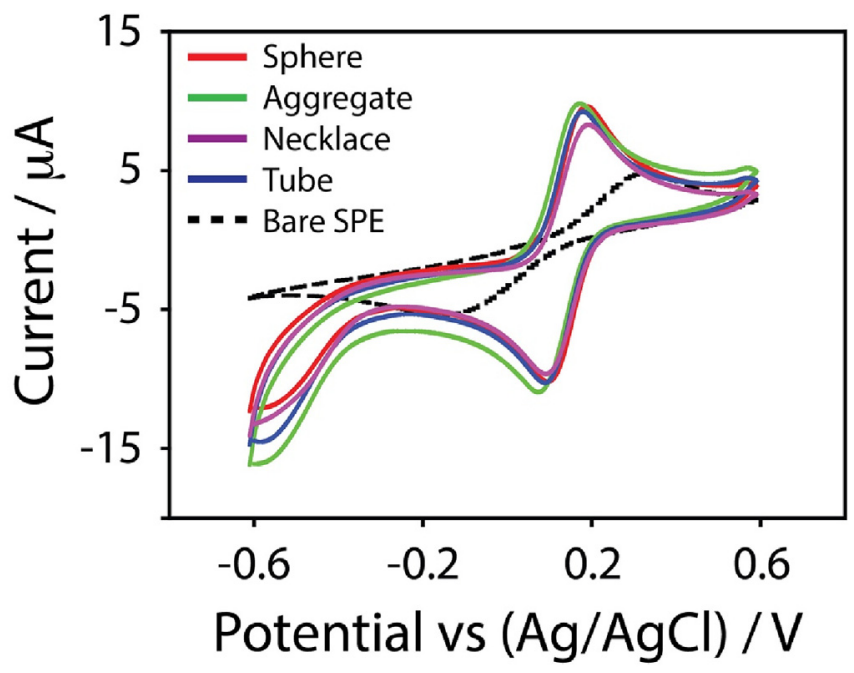

Fig. 4. Cyclic voltammograms recorded in presence of $1 \mathrm{mM}$ of ferro/ferricyanide in $0.05 \mathrm{M}$ phosphate buffer containing $0.1 \mathrm{M}$ potassium chloride ( $\mathrm{pH} 7.4)$. A) All the cyclic voltammograms were recorded at scan rate of $50 \mathrm{mV} / \mathrm{s}$ using bare SPE (dashed line) or SPE modified with magnetic CSs-based structures, i.e. sphere (red line), aggregate (green line), necklace (violet line), tube (blue line). (For interpretation of the references to color in this figure legend, the reader is referred to the Web version of this article.)
As an example, in Fig. 4 we reported the electrochemical responses using SPEs modified with sphere, aggregate, necklace, and tube structures in respect to the bare SPE at scan rate of $50 \mathrm{mV} / \mathrm{s}$, to shed light the peak-to-peak separation and resolution of the peaks in the case of modified SPEs. It is interesting to observe how these modifiers compare very favorably with the others carbonaceous materials that have been utilized as electrode modifiers, i.e. graphene oxide, reduced-graphene oxide, carbon nanotubes, as reported by our and others research groups [22-25].

\subsection{Cyclic voltammetric study of modified-SPEs}

With the aim of evaluating the CSs-based structures sensing properties, the different modified electrodes were tested towards relevant analytes in biomedical field namely ascorbic acid, dopamine, cysteine, serotonin, and NADH. In Fig. 5 the cyclic voltammograms related to the above cited species are displayed. From the results reported, it comes out that the change in the shape and complexity of the CSs-based structures shows a different electrochemical behaviour. The voltammograms in Fig. 5 clearly evidence the enhancement of electrochemical behaviour of the SPEs modified with magnetic CSs-based structures when compared with the voltammograms obtained using bare SPE. In order to better understand the outcomes, all the overpotentials were reported in Table 2. In detail, for all studied species, the overpotentials appear lower than those observed at the unmodified electrode, owing to the presence of modifiers and comparable with the ones obtained using SPEs modified with nanomaterials namely carbon black and carbon nanotubes [22].

Although all structures displayed a decrease of the overpotential, they are characterized by slight differences which could be dependent on their specific shapes. This slight different electrochemical behaviour is ascribed to some key factors including the presence of iron nuclei, the defective sites of the carbon skeleton, and the outer surface roughness. For instance, the tubes structures (Fig. 1D) mainly exploit the highest overpotentials with respect to the other structures, considering all the species analyzed, e.g. cysteine needs of $0.68 \mathrm{~V}$ ( $\mathrm{vs} \mathrm{Ag} / \mathrm{AgCl}$ ) to be oxidized at tubesmodified SPE, while when sphere (Fig. 1A), aggregate (Fig. 1B) or necklace (Fig. 1C) are adopted as SPE modifier, the oxidation potential is $0.52,0.54$, and $0.51 \mathrm{~V}$, respectively. This behaviour might 

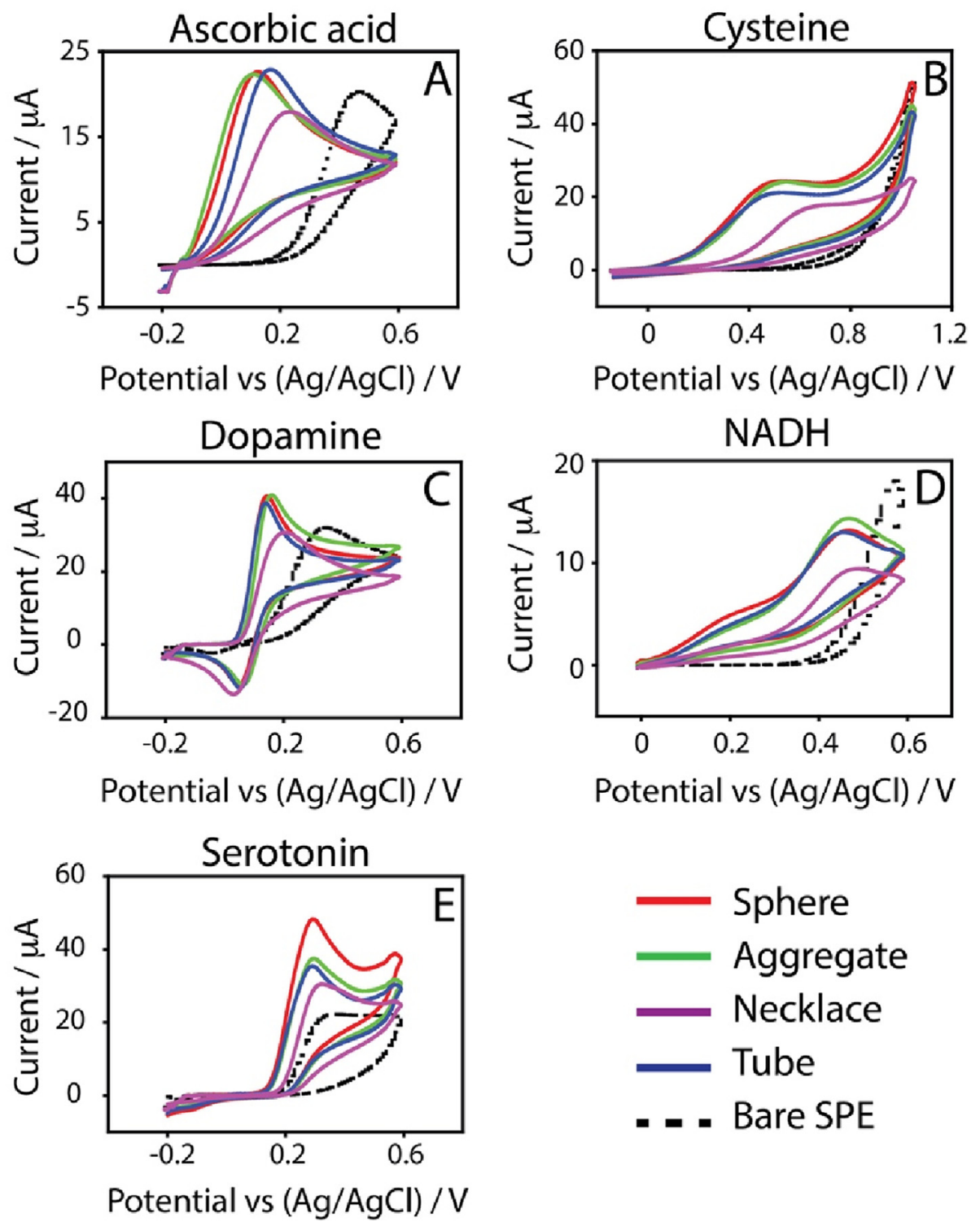

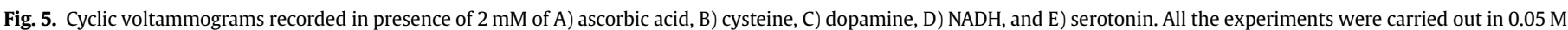

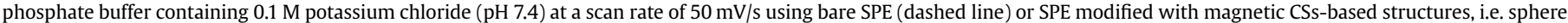

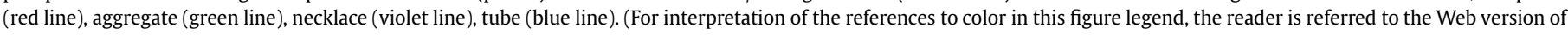
this article.)

Table 2

Overpotentials expressed in $\mathrm{V}$ ( $\mathrm{vs} \mathrm{Ag} / \mathrm{AgCl}$ ) for the species detected at different modified-SPEs.

\begin{tabular}{lccccc}
\hline Species/SPE & Bare & Sphere & Aggregate & Necklace & Tube \\
\hline Ascorbic acid & $0.47 \mathrm{~V}$ & $0.12 \mathrm{~V}$ & $0.10 \mathrm{~V}$ & $0.17 \mathrm{~V}$ & $0.23 \mathrm{~V}$ \\
Dopamine (Ox) & $0.34 \mathrm{~V}$ & $0.14 \mathrm{~V}$ & $0.17 \mathrm{~V}$ & $0.14 \mathrm{~V}$ & $0.22 \mathrm{~V}$ \\
Dopamine (Red) & $-0.05 \mathrm{~V}$ & $0.06 \mathrm{~V}$ & $0.06 \mathrm{~V}$ & $0.05 \mathrm{~V}$ & $0.03 \mathrm{~V}$ \\
Cysteine & $0.80 \mathrm{~V}$ & $0.52 \mathrm{~V}$ & $0.54 \mathrm{~V}$ & $0.51 \mathrm{~V}$ & $0.68 \mathrm{~V}$ \\
NADH & $0.57 \mathrm{~V}$ & $0.46 \mathrm{~V}$ & $0.47 \mathrm{~V}$ & $0.44 \mathrm{~V}$ & $0.47 \mathrm{~V}$ \\
Serotonin & $0.36 \mathrm{~V}$ & $0.29 \mathrm{~V}$ & $0.29 \mathrm{~V}$ & $0.30 \mathrm{~V}$ & $0.32 \mathrm{~V}$ \\
\hline
\end{tabular}

be ascribed to the less defective surface that decreases the number of reactive sites. Instead, the other structures have similar overpotentials. However, the presence of iron nuclei would be considered the main responsible of the effects in improving electrochemistry of printed platforms. The iron nuclei impurities in the magnetic CSs-structures act as nanospot centers for redox reaction, as reported in other systems, i.e. dopamine at SWCNTs/ iron (III) oxide, hydrogen peroxide at ferric hexacyanoferrate (Prussian Blue), oxygen at graphene-iron composite [26-28]. Again, the absence of iron nuclei (as in the case of the non-magnetic aggregate structures) leads only to a negligible electrocatalysis improvement in respect to the bare SPE [14], allowing for a predominant effect of iron nuclei over the defect sites.

\subsection{Amperometric study of modified-SPES}

The electroanalytical performances of SPEs modified with CSsbased structures were evaluated by means of amperometric batch analysis using ascorbic acid as selected analyte and $150 \mathrm{mV}$ as an applied potential. Fig. 6a illustrates the amperograms obtained at the concentration range from 0 to $150 \mu \mathrm{M}$ in phosphate buffer, while Fig. $6 \mathrm{~b}$ the calibration curves, showing that SPE modified with aggregate structures is characterized by highest sensitivity 


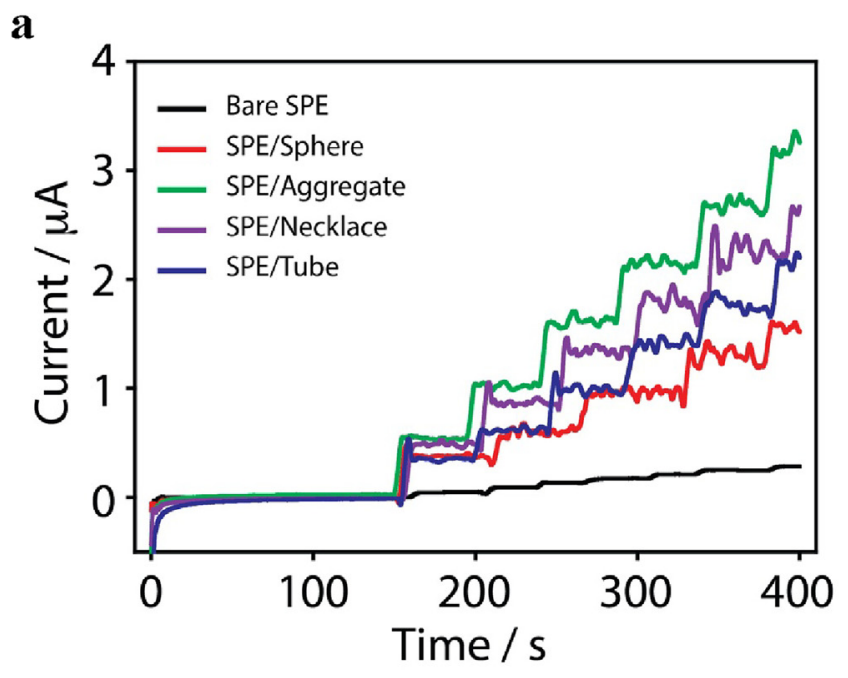

b

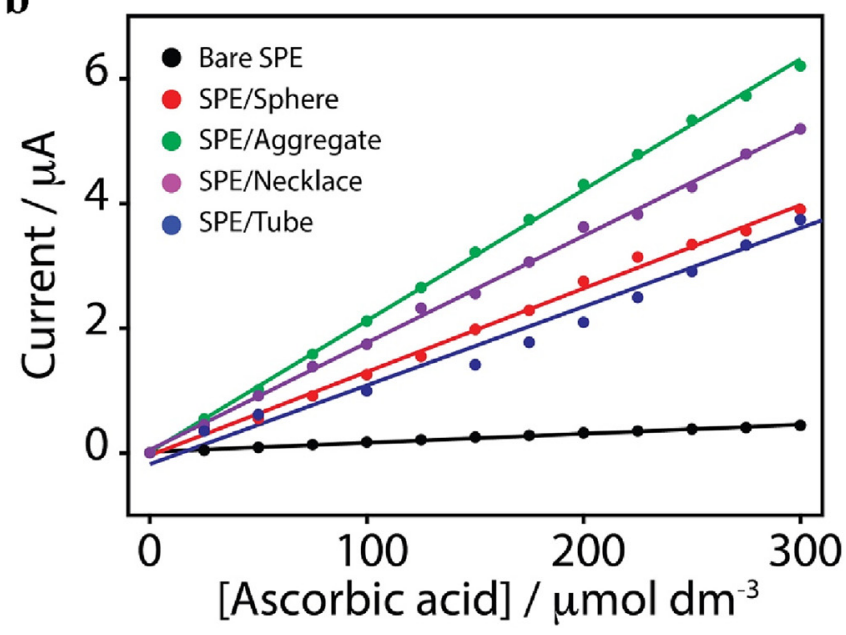

Fig. 6. Amperograms (a) and calibration curves (b) obtained in phosphate buffer $0.05 \mathrm{M}+\mathrm{KCl} 0.1 \mathrm{M}, \mathrm{pH}=7.4$ to successive additions $(25 \mu \mathrm{M})$ of ascorbic acid using bare SPE (dashed line) or SPE modified with magnetic CSs-based structures, i.e. sphere (red line), aggregate (green line), necklace (violet line), tube (blue line). (For interpretation of the references to color in this figure legend, the reader is referred to the Web version of this article.)

followed by SPE modified with necklace, tube and sphere structures. In detail, in Table 3 sensitivities and limit of detection (LOD) obtained by using SPEs modified with different CSs-based structures were reported. The modified SPEs showed an improvement of the analytical performances compare to the bare SPE due to the presence of the CSs structures on the working electrode surface. The different sensitivity obtained using SPEs modified with different CSs is due to the different morphologies of the structures, in detail SPE modified with aggregate, necklace and tube are characterized by higher sensitivity in respect to SPE modified with sphere. This behaviour is ascribed to their continuous structures in the length, boosting the electron conduction. Among the elongated structures, SPE modified with aggregate structures gives the best analytical performances, probably due to the lower size of the sphere precursor (diameter equal to $175 \pm 10 \mathrm{~nm}$ ) combined with most ordered internal structures [14], thus this type of structure was selected for the further studies.

\subsection{Exploitation of magnetic properties of CSs}

It seems clear how the presence of the CSs-based modifiers allows for improving the electrochemical behaviour of the printed electrodes, both in terms of sensitivity and overpotential, with respect to the bare platform. Furthermore, the presence of iron nuclei in the above-cited structures confers to the CSs-based assemblies (i.e. aggregate structures) a clear magnetic feature. In Fig. 7, a net example of the magnetic behaviour is reported. In this case, it is shown how the aggregate structures can be easily attracted by an external magnet, exploiting their unique characteristics. In principle, these magnetic structures might be used separately from a SPE, for example for adsorbing species that are present in a sample; successively they can be re-concentrated on the SPE in order to carry out the detection, by exploiting their magnetic properties, as described in Fig. 8.

Compared with existing technology based on the magnetic beads, which are widely used in the development of analytical assays i.e. immunologic, the magnetic CSs-based structures might be involved for the development of novel multi-task devices. In the previous section, their electroanalytical effectiveness has been proven. However, their relevance should not be focused solely over the possibility to improve the performance of SPE: to do this, a plethora of materials (not only carbon-based) can be taken into account. The real added valued of these structures is that they are capable to accomplish three assignments, within the same object: 1) to sample a target molecule, 2) to be magnetically gathered on any substrate, and 3) to sense the "sampled" analyte. This represents the most beneficial role that can be attributed to these objects. In order to highlight this possibility, the magnetic aggregate structures displayed interesting adsorbing features. More precisely, ferricyanide, fuchsine, methylene blue, and Prussian blue, were taken as model targets to be adsorbed and released. To carry out these experiments $1 \mathrm{mg}$ of magnetic aggregate structures was immersed in a $3 \mathrm{~mL}$ solution containing one of the above-cited species. After $10 \mathrm{~min}$, the sample was adsorbed on the aggregate structures, dried, and magnetically concentrated on the white spot. A $10 \mu \mathrm{L}$-drop of water was used to evaluate the release of the adsorbed molecules. As illustrated in Fig. 8A, the CSs-based structures were stirred for $10 \mathrm{~min}$ in a flask, containing the sample to be collected. Successively, the aggregate structures (with the adsorbed molecules) were separated from the sample-containing flask with an external magnet, dried, and deposited onto a white substrate, i.e. filter paper. As showed in Fig. 8B, an external magnet was capable to move the magnetic CSs from a white spot to another. Thanks to the magnetic guidance, the aggregate structures remained fixed on a precise spot. After a $10 \mu \mathrm{L}$-drop of water has been added to the magnetic structures, the adsorbed species were clearly released to the paper, evidenced with a change of color depending on the released molecules (Fig. $8 \mathrm{C}$ ), demonstrating the relevant role in collecting and releasing molecules. Successively, preliminary

Table 3

Sensitivities and LOD for the tested compounds at CSs-modified SPEs using amperometric batch analysis.

\begin{tabular}{|c|c|c|c|c|c|}
\hline & Bare SPE & Sphere-SPE & Aggregate-SPE & Necklace-SPE & Tube-SPE \\
\hline LOD $(\mathrm{mM})$ & 2 & 4 & 3 & 5 & 5 \\
\hline Sensitivity $\left(\mathrm{A} / \mathrm{M} \mathrm{\textrm {cm } ^ { 2 } )}\right.$ & 0.02 & 0.19 & 0.30 & 0.24 & 0.17 \\
\hline
\end{tabular}




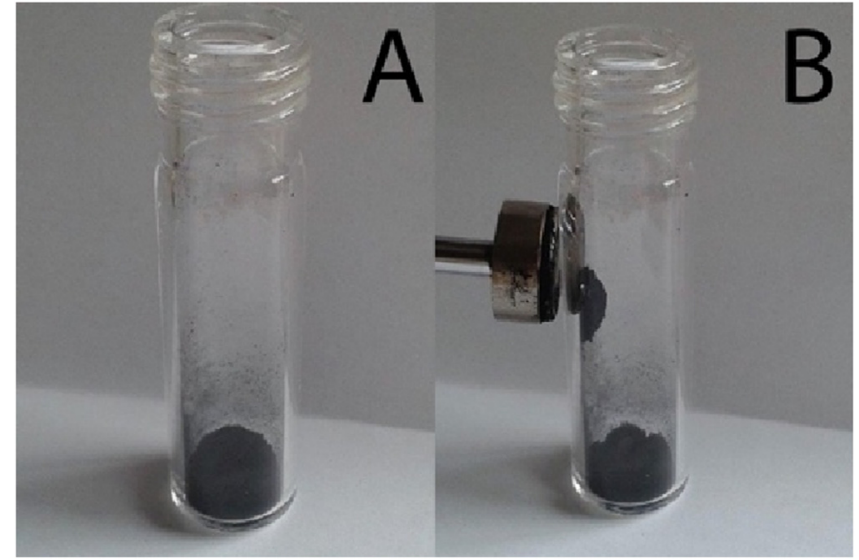

Fig. 7. Photograph of aggregate structures before (A) and during (B) the action of an external magnet.

experiments were performed by collecting and detecting ferricyanide electrochemically. To carry out this approach, the magnetic aggregates were immersed in the sample solution, freely to move. They were stirred in a flask where the molecule to sample, i.e. ferricyanide, was present. Successively, the aggregate structures (with the adsorbed ferricyanide) were separated from the samplecontaining flask with an external magnet, dried, and deposited onto the three-configured SPE, as displayed in Fig. 9A. Lastly, the CSs-SPE was covered with a $50 \mu \mathrm{L}$-drop of phosphate buffer in order to allow the analyte to be detected. Fig. 9B displays the

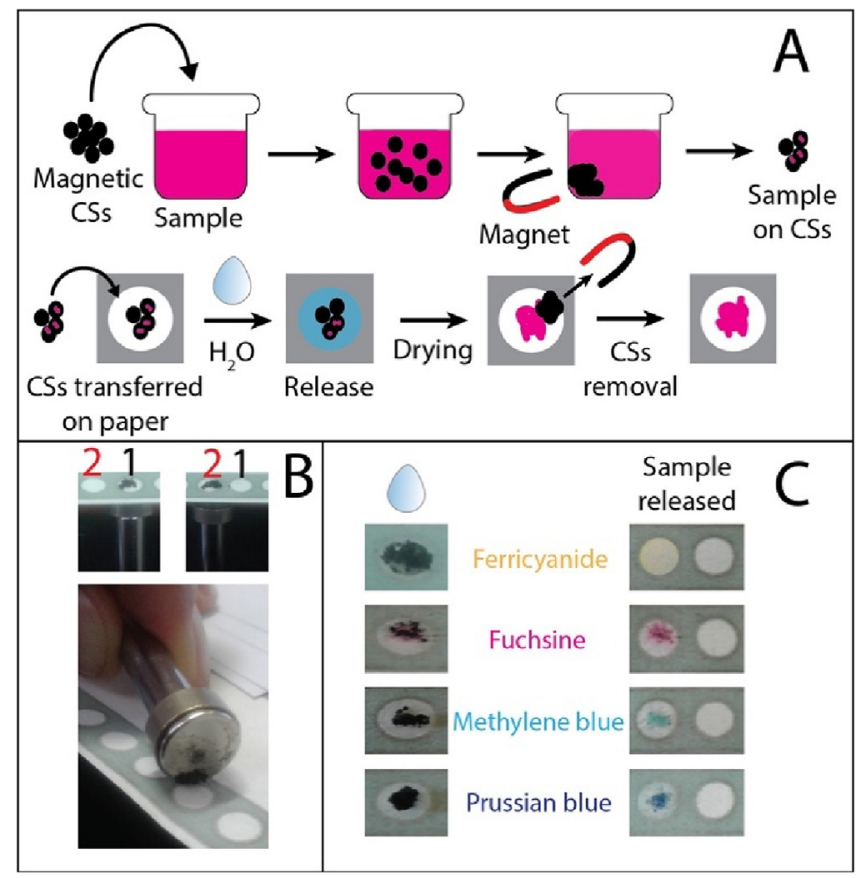

Fig. 8. A) A schematic representation of the experimental path followed to sample a generic molecule on the aggregate magnetic structures, and successively released on the paper-based substrate, i.e. filter paper. B) Photographs regarding the capacity of the aggregate structures to be magnetically driven and concentrated onto a substrate. $C$ ) Release of the adsorbed ferricyanide $(10 \mathrm{mM})$, fuchsine $(0.1 \mathrm{mg} / \mathrm{mL})$, methylene blue $(0.1 \mathrm{mg} / \mathrm{mL})$, and Prussian blue $(0.1 \mathrm{mg} / \mathrm{mL})$. To carry out these experiments $1 \mathrm{mg}$ of magnetic aggregate structures have been immersed in a $3 \mathrm{~mL}$ solution containing one of the above-cited species. After $10 \mathrm{~min}$, the sample has been adsorbed on the aggregate structures, dried, and magnetically concentrated on the white spot. A $10 \mu \mathrm{L}-$ drop of water was used to evaluate the release of the adsorbed molecules.

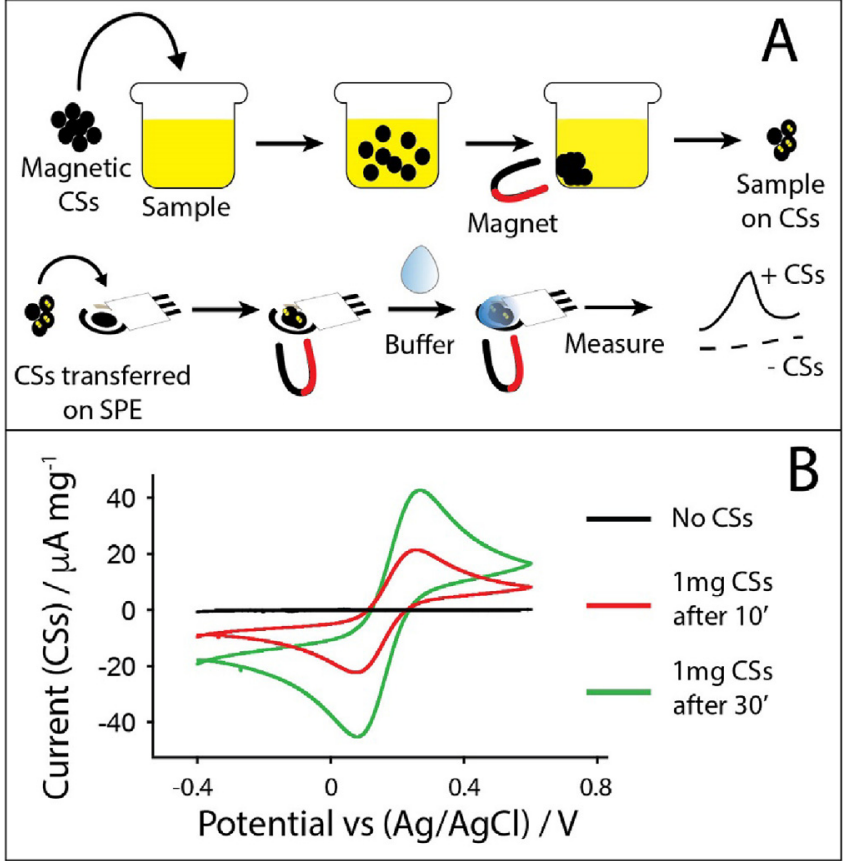

Fig. 9. A) A schematic representation of the experimental path followed to collect a ferricyanide on the aggregate CSs and release the same molecule on a printed electrode, i.e. SPE. B) Electrochemical detection by using a bare SPE without aggregate CSs (black line) and in presence of $1 \mathrm{mg}$ of aggregate CSs that have been immersed for 10 (red line) and 30 (green line) $\mathrm{min}$ in a $3 \mathrm{~mL}$ solution containing $10 \mathrm{mM}$ ferricyanide. The SPEs were covered with a $50 \mu \mathrm{L}$-drop of phosphate buffer prior the measurement was started. Cyclic voltammograms have been carried out at a scan rate of $50 \mathrm{mV} / \mathrm{s}$. (For interpretation of the references to color in this figure legend, the reader is referred to the Web version of this article.)

voltammograms obtained by exploiting the properties of the CSs structures, utilizing different "sampling" periods, i.e. 10 and $30 \mathrm{~min}$, and allowing an increasing of sensitivity at the increase of the sampling time. From these results, the analyte is sampled from the chosen solution and it is readily detected at the printed electrode, just taking advantage of a completely integrated smart material.

\section{Conclusions}

In summary, this study provided an effective application in the electroanalytical field by interfacing magnetic carbon spheres with screen-printed electrodes as a model system. Different architectures have been characterized both morphologically and electrochemically. Through easy drop casting method, screen-printed electrodes have been efficiently modified with carbonaceous structures, which conferred to the electrodes a clear electrochemical enhancement, regarding the detection of relevant species such as ferro/ferricyanide, ascorbic acid, dopamine, cysteine, serotonin, and NADH. The structures used to modify SPEs confer tuned electroanalytical properties due to the different morphologies, since SPEs modified with one dimensional materials (ie. aggregate, necklace and tube) are characterized by higher sensitivity in respect to SPE modified with sphere (zero dimensional material).

These magnetic microsized structures, thanks to their adsorbing properties and also to the presence of iron nuclei within the carbonaceous lattice, allowed for the development of a novel conceptual step forward in the field of sensing (and not only). The exploitation of their adsorptive, magnetic, and electrocatalytic features, offers the possibility to develop a novel analytical research line: these CSs-based magnetic structures can sample particular analytes and then being coupled with electrochemical external 
devices for detection. In this work, the advantages of these CSsbased products were positively demonstrated via preliminary efforts by combining them with printed electrodes. We are confident that these magnetic materials, assisted by the CVD synthetic approach, will be capable to provide the end-users an all-in-one platform, that has been demonstrated to be easily interfaced with sensing devices. Besides the promising results reached in this study, these smart materials might be coupled to biomolecules, i.e. enzymes, antibodies, in order to develop specific bioassays. For future studies, these smart materials will be functionalized with biomolecules to perform more targeted biomedical and environmental assignments.

\section{Acknowledgements}

S.C. acknowledges Fondazione Umberto Veronesi for PostDoctoral Fellowship 2018. M.S. acknowledges the European Community for the RISE Project CoExAN GA644076. F.A. acknowledges the ERANETMED Project NanoSWS. M.S. and F.A. acknowledge the University of Rome "Tor Vergata" for the NANOSPES project. The authors acknowledge Prof. M. Passacantando, University of L'Aquila, for the Raman measurements and Prof. I. Cacciotti, University of Rome "Niccolò Cusano" for SEM measurements.

\section{References}

[1] A.A. Deshmukh, S.D. Mhlanga, N.J. Coville, Carbon spheres, Mater. Sci. Eng. R Rep. 70 (2010) 1-28.

[2] M.S. Dresselhaus, G. Dresselhaus, P.C. Eklund, Science of Fullerenes and Carbon Nanotubes, Academic Press, New York, 1996.

[3] L. Dai, Carbon Nanotechnology: Recent Developments in Chemistry, Physics, Materials Science and Device Applications, Elsevier, New York, 2006.

[4] S. Wang, H. Sun, H.M. Ang, M.O. Tadé, Adsorptive remediation of environmental pollutants using novel graphene-based nanomaterials, Chem. Eng. J. 226 (2013) 336-347.

[5] W. Lv, Z. Li, Y. Deng, Q.H. Yang, F. Kang, Graphene-based materials for electrochemical energy storage devices: opportunities and challenges, Energy Storage Mater. 2 (2016) 107-138.

[6] S. Cinti, F. Arduini, Graphene-based screen-printed electrochemical (bio) sensors and their applications: efforts and criticisms, Biosens. Bioelectron. 89 (2017) 107-122.

[7] C.B. Jacobs, M.J. Peairs, B.J. Venton, Carbon nanotube based electrochemical sensors for biomolecules, Anal. Chim. Acta 662 (2010) 105-127.

[8] M. Pumera, A. Ambrosi, A. Bonanni, E.L.K. Chng, H.L. Poh, Graphene for electrochemical sensing and biosensing, TrAC 29 (2010) 954-965.

[9] L. Kong, X. Lu, X. Bian, W. Zhang, C. Wang, Accurately tuning the dispersity and size of palladium particles on carbon spheres and using carbon spheres/ palladium composite as support for polyaniline in $\mathrm{H}_{2} \mathrm{O}_{2}$ electrochemical sensing, Langmuir 26 (2010) 5985-5990.

[10] X. Bo, J. Bai, B. Qi, L. Guo, Ultra-fine Pt nanoparticles supported on ionic liquid polymer-functionalized ordered mesoporous carbons for nonenzymatic hydrogen peroxide detection, Biosens. Bioelectron. 28 (2011) 77-83.

[11] X. Bian, K. Guo, L. Liao, J. Xiao, J. Kong, C. Ji, B. Liu, Nanocomposites of palladium nanoparticle-loaded mesoporous carbon nanospheres for the electrochemical determination of hydrogen peroxide, Talanta 99 (2012) 256-261.

[12] S.P. Dubey, A.D. Dwivedi, I.-C. Kim, M. Sillanpaa, Y.-N. Kwon, C. Lee, Synthesis of graphene-carbon sphere hybrid aerogel with silver nanoparticles and its catalytic and adsorption applications, Chem. Eng. J. 244 (2014) 160-167.

[13] P.M. Visakh, M. Morlanes, Nanomaterials and Nanocomposites: Zero-to Threedimensional Materials and Their Composites, John Wiley \& Sons, 2016.

[14] M. Scarselli, F. Limosani, M. Passacantando, F. D'Orazio, M. Nardone, I. Cacciotti, E. Gautron, F. Arduini, M. De Crescenzi, Adv. Mater. Interfaces (2018), https://doi.org/10.1002/admi.201800070.

[15] A. Abouelsayed, B. Anis, S. Hassaballa, A.S. Khalil, U.M. Rashed, K.A. Eid, E. AlAshkar, Preparation, characterization, Raman, and terahertz spectroscopy study on carbon nanotubes, graphene nano-sheets, and onion like carbon materials, Mater. Chem. Phys. 189 (2017) 127-135.

[16] P. Serp, R. Feurer, P. Kalck, Y. Kihn, J.L. Faria, J.L. Figueiredo, A chemical vapour deposition process for the production of carbon nanospheres, Carbon 39 (2001) 621-626.

[17] S. Cinti, F. Santella, D. Moscone, F. Arduini, $\mathrm{Hg}^{2+}$ detection using a disposable and miniaturized screen-printed electrode modified with nanocomposite carbon black and gold nanoparticles, Environ. Sci. Pollut. Res. 23 (2016) 8192-8199.

[18] S. Cinti, D. Neagu, M. Carbone, I. Cacciotti, D. Moscone, F. Arduini, Nove carbon black-cobalt phthalocyanine nanocomposite as sensing platform to detect organophosphorus pollutants at screen-printed electrode, Electrochim. Acta 188 (2016) 574-581.

[19] X. Ji, C.E. Banks, A. Crossley, R.G. Compton, Oxygenated edge plane sites slow the electron transfer of the ferro-/ferricyanide redox couple at graphite electrodes, ChemPhysChem 7 (2006) 1337-1344.

[20] R.G. Compton, C.E. Banks, Understanding Voltammetry, World Scientific, Singapore, 2007.

[21] R.S. Nicholson, Theory and application of cyclic voltammetry for measurement of electrode reaction kinetics, Anal. Chem. 37 (1965) 1351-1355.

[22] S. Cinti, F. Arduini, M. Carbone, L. Sansone, I. Cacciotti, D. Moscone G. Palleschi, Screen-printed electrodes modified with carbon nanomaterials: a comparison among carbon black, carbon nanotubes and graphene, Electroanal 27 (2015) 2230-2238.

[23] A. Martin, A. Escarpa, Graphene: the cutting-edge interaction between chemistry and electrochemistry, Trends Anal. Chem. 56 (2014) 13-26.

[24] R.R. Moore, C.E. Banks, R.G. Compton, Basal plane pyrolytic graphite modified electrodes: comparison of carbon nanotubes and graphite powder as electrocatalysts, Anal. Chem. 76 (2004) 2677-2682.

[25] F. Campanha Vicentini, A.E. Ravanini, L.C.S. Figueiredo-Filho, C.E. Banks, O. Fatibelo-Filho, Imparting improvements in electrochemical sensors: evaluation of different carbon blacks that give rise to significant improvement in the performance of electroanalytical sensing platforms, Electrochim. Acta 157 (2015) 125-133.

[26] A.S. Adekunle, B.O. Agboola, J. Pillay, K.I. Ozoemena, Electrocatalytic detection of dopamine at single-walled carbon nanotubes-iron (III) oxide nanoparticles platform, Sens. Actuators B 148 (2010) 93-102.

[27] S. Cinti, F. Arduini, D. Moscone, G. Palleschi, L. Gonzalez-Macia, A.J. Killard, Cholesterol biosensor based on inkjet-printed Prussian blue nanoparticlemodified screen-printed electrodes, Sens. Actuators B 221 (2015) 187-190.

[28] K. Parvez, S. Yang, Y. Hernandez, A. Winter, A. Turchanin, X. Feng, K. Müllen, Nitrogen-doped graphene and its iron-based composite as efficient electrocatalysts for oxygen reduction reaction, ACS Nano 6 (2012) 9541-9550. 